\title{
Isolation of Nicotiana plumbaginifolia cDNAs encoding isoforms of serine acetyltransferase and $O$-acetylserine (thiol) lyase in a yeast two-hybrid system with Escherichia coli cysE and cysK genes as baits ${ }^{\star \bullet}$
}

\author{
Frantz Liszewska ${ }^{1}$, Dali Gaganidze ${ }^{2}$ and Agnieszka Sirko ${ }^{1 凶}$ \\ ${ }^{1}$ Institute of Biochemistry and Biophysics, Polish Academy of Sciences, Warszawa, Poland; \\ ${ }^{2}$ Scientific Research Center of Agriculture Biotechnology, Tibilisi, Georgia
}

Received: 23 December, 2004; accepted: 03 March, 2005

Key words: cDNA cloning, cysteine biosynthesis, genome walking, tobacco, yeast two-hybrid system

\begin{abstract}
We applied the yeast two-hybrid system for screening of a cDNA library of Nicotiana plumbaginifolia for clones encoding plant proteins interacting with two proteins of Escherichia coli: serine acetyltransferase (SAT, the product of cysE gene) and $O$-acetylserine (thiol) lyase A, also termed cysteine synthase (OASTL-A, the product of cysK gene). Two plant cDNA clones were identified when using the cysE gene as a bait. These clones encode a probable cytosolic isoform of OASTL and an organellar isoform of SAT, respectively, as indicated by evolutionary trees. The second clone, encoding SAT, was identified independently also as a "prey" when using cysK as a bait. Our results reveal the possibility of applying the two-hybrid system for cloning of plant cDNAs encoding enzymes of the cysteine synthase complex in the
\end{abstract}

\footnotetext{
Financial support by the State Committee for Scientific Research (KBN; Poland, grant No. 6P04A07121) and by the EU commission through funding of FP5 projects QLRT-2000-00103 and QLRT-2001-00429 as well as the Centre of Excellence in Molecular Biotechnology-Getting European Dimension (ICA-CT-2000-70010) is greatly acknowledged. D.G. was a holder of a fellowship from the European Fellowships Fund at IBB PAS.

-Accesssion numbers: The sequences of the N. plumbaginifolia sat1 and oastl (cs1) cDNAs and the sequence of a genomic DNA fragment located upstream of sat1 have been deposited in the EMBL database under accession numbers AY450296, AY450295 and AY898624, respectively.

${ }^{\square}$ Correspondence to: A. Sirko, Institute of Biochemistry and Biophysics, Polish Academy of Sciences, A. Pawińskiego 5A, 02-106 Warszawa, Poland; tel.: (48 22) 658 4801; fax: (48 22) 658 4804; e-mail: asirko@ibb.waw.pl
}

Abbreviations: At, Arabidopsis thaliana; CS, cysteine synthase, also called OASTL, $O$-acetylserine (thiol) lyase (EC 2.5.1.47, previously EC 4.2.99.8); ORF, open reading frame; OAS, $O$-acetylserine; O/N, over night; NP, Nicotiana tabacum; SAT, serine acetyltransferase (EC 2.3.1.30). 
two-hybrid system. Additionally, using genome walking sequences located upstream of the sat1 cDNA were identified. Subsequently, in silico analyses were performed aiming towards identification of the potential signal peptide and possible location of the deduced mature protein encoded by sat1.

Many steps of the sulfur assimilation pathway are conserved among bacteria, fungi and plants (Kredich, 1996; Marzluf, 1997; Leustek \& Saito, 1999; Brzywczy et al., 2002; Takagi et al., 2003). Two enzymes, serine acetyltransferase (SAT; EC 2.3.1.30) and $O$-acetylserine (thiol) lyase (OASTL; EC 2.5.1.47) are responsible for conducting the two final steps of the cysteine biosynthesis pathway, namely (i) production of $O$-acetylserine (OAS) from serine and acetyl coenzyme A and (ii) incorporation of sulfide into OAS resulting in cysteine production, respectively. SAT and OASTL from Escherichia coli and Salmonella typhimurium have been intensively studied and are well characterized (Burkhard et al., 1998; Hindson \& Shaw, 2003; Tai et al., 2001). In plants, several genes for each of the two enzymes exist (for a recent review see Hell et al., 2002). cDNAs from plants encoding various SAT and OASTL isoenzymes have been isolated. Location of these enzymes in various plant compartments (plastids, mitochondria and cytosol) is well documented (Kuske et al., 1996; Noji et al., 1998; Jost et $a l ., 2000)$. SAT and OASTL can form a bi-enzymatic complex (also called the cysteine synthase complex). This process plays an important regulatory role in the cysteine biosynthesis pathway. Molecular interactions between the enzymes forming the complex were studied in vitro for both bacterial (Mino et al., 2000) and plant (Berkowitz et al., 2002; Droux et al., 1998) enzymes. Moreover, mixed interactions between plant isoenzymes from various compartments were shown in the yeast two-hybrid system (Bogdanova \& Hell, 1997). However, heterologous bacterial-plant interactions have only been observed in vitro, in gel-filtration experiments with protein extracts from bacteria transformed with plant cDNA encoding SAT (Droux et al., 1998).
Functional substitution of the bacterial enzymes by their plant counterparts is possible and this fact has been successfully employed for cloning of plant cDNAs encoding SAT (Saito et al., 1995; Howarth et al., 1997; Wirtz \& Hell, 2003) and OASTL (Noji et al., 1994) due to their ability to complement functionally the respective $E$. coli cysteine auxotrophic mutants. On the other hand, at least two research groups, including ours, have reported expression of bacterial genes encoding SAT and OASTL in plants (Blaszczyk et al., 1999; 2002; Harms et al., 2000; Liszewska et al., 2001; Sirko et al., 2004). The ability to form in vivo the mixed bacterial-plant complexes might be a significant factor affecting the total yield of the reactions catalyzed by the SAT and OASTL enzymes in such transgenic plants. However, the problem of potential interactions of these bacterial enzymes with plant proteins has not been explored sufficiently. Therefore, we decided to screen an Nicotiana plumbaginifolia cDNA library for clones encoding proteins involved in heterologous interactions with bacterial SAT and OASTL using the yeast two-hybrid system (Fields \& Song, 1989).

\section{MATERIALS AND METHODS}

Bacterial strains. The standard E. coli strains (DH5 $\alpha$, XL1Blue) used for cloning and plasmid amplification were grown in LB with ampicillin (100 $\mathrm{mg} \mathrm{l}^{-1}$ ). For the selective rescue of appropriate plasmids extracted from yeasts after cDNA library screenings the E. coli KC8 strain (Clontech) was grown on solid M9 minimal medium (Sambrook et al., 1989) supplemented with thiamine-HCl (0.5 $\mathrm{mg} \mathrm{l}^{-1}$ ), histidine $\left(20 \mathrm{mg} \mathrm{l}^{-1}\right)$, and either leucine $\left(100 \mathrm{mg} \mathrm{l}^{-1}\right.$, for rescue of the DNA- 
binding domain fusion plasmids) or tryptophan $\left(20 \mathrm{mg}^{-1}\right.$, for rescue of the activation domain fusion plasmids).

Yeast two-hybrid screening. The Nicotiana plumbaginifolia cDNA library was a kind gift of Dr. Witold Filipowicz, Friedrich Miescher Institute for Biomedical Research (Basel, Switzerland). Yeast plasmids pGBT9 and pGAD424 were from Clontech; plasmid pCL1 (Fields \& Song, 1989) containing the entire GAL4-encoding sequence, was used as a positive control. Manipulation of yeast cells and library screening were carried out according to standard protocols (Clontech Yeast Protocol Handbook, PT3024-1). The Saccharomyces cerevisiae strain PJ69-4 (James et al., 1996) was used in all experiments.

DNA methods, oligonucleotides used. All restriction enzymes (MBI Fermentas) and T4 DNA ligase (Promega) were used under conditions recommended by the suppliers. Conventional techniques were used for DNA manipulation and transformation (Sambrook et al., 1989). The genes cysE and cysK were amplified by PCR using genomic DNA of $E$. coli K-12 as a template, Pfu polymerase (Stratagene), and the following pairs of primers: 5'-CGG AAT TCA TGT CGT GTG AAG AAC TGG AAA-3' and 5'-CGG TCG ACT TAG ATC CCA TCC CCA TAC TCA-3' for cysE, and 5'-CGG AAT TCA TGA GTA AGA TTT TTG AAG-3' and $5^{\prime}$-CGG TCG ACG CTG GCA TTA CTG TGC-3' for cysK. The PCR products, $0.8 \mathrm{~kb}$ (cysE) and $1.0 \mathrm{~kb}$ (cysK) were subsequently cloned into pGEM-T easy vector (Promega). The final plasmids: pCE-GBT and pCE-GAD (containing cysE) and pCK-GBT and pCK-GAD (containing cysK) were obtained by sub-cloning of the respective EcoRI-EcoRI inserts from the pGEM-T easy-based plasmids into pGBT9 and pGAD424, respectively.

$\beta$-galactosidase assay. $\beta$-Galactosidase liquid culture assay using o-nitrophenyl $\beta$-D-galactopyranoside (ONPG) as a substrate was performed according to the procedure described in the Clontech Yeast Protocols Handbook, PT3024-1. One unit of $\beta$-galactosidase activity is defined as the amount of enzyme which hydrolyzes $1 \mu$ mole of ONPG to $O$-nitrophenol and D-galactose per minute per cell. $\beta$-Galactosidase activity in yeast grown on plates was visualized with $\mathrm{X}-\alpha$-Gal (Calbiochem) as a substrate.

Cysteine synthase assay. Yeast extracts were prepared from $30 \mathrm{ml}$ yeast cultures in SD-TL liquid medium grown over night $(\mathrm{O} / \mathrm{N})$ at $28^{\circ} \mathrm{C}$ with shaking. Cells, suspended in $100 \mathrm{mM}$ Tris/HCl pH 7.5 with addition of Complete ${ }^{\circledR}$ proteases inhibitors (Roche), were mechanically disrupted using acidwashed glass beads (425-600 mesh, Sigma). Protein concentrations in the extracts were determined using Protein Assay Kit (BioRad) and bovine serum albumin as a standard. OASTL activity in the yeast extracts was assayed by monitoring the formation of L-cysteine from OAS and sulfide after addition of $5 \mu \mathrm{l}$ yeast extract to $145 \mu \mathrm{l}$ reaction mixture containing: $100 \mathrm{mM}$ Tris/ $\mathrm{HCl}$ $\mathrm{pH}$ 7.5, $100 \mathrm{mM}$ dithiothreitol (DTT), $8 \mathrm{mM}$ OAS and $3 \mathrm{mM}$ sodium sulfide. After $5 \mathrm{~min}$ incubation at room temperature, L-cysteine content in the mixture was measured spectrophotometrically, according to a procedure adapted from the Gaitonde's method (Gaitonde, 1967). One unit of OASTL activity is defined as the amount of enzyme which catalyzes the formation of 1 nmole of L-cysteine per minute under the conditions of the assay.

Genome walking. Construction of genome walking libraries and DNA fragment amplification were performed using the GenomeWalker ${ }^{\circledR}$ Universal Kit (Clontech-BD Biosciences) and gene-specific reverse outer and nested primers designed for this purpose (PSATo and PSATn). The PSATo (5'-GTC GAT TTG GGG CTT GTT TGG ATC AC-3') and PSATn (5'-GTG GAT TTG TAG GAG GAG TTT TGG TT- $3^{\prime}$ ) primers were designed according to recommendations and suggestions of the manufacturer.

Computer analysis. Similarity searches were performed with tblastn, blastp and blastx at NCBI [http://www.ncbi.nlm.nih.gov/ 
BLAST/]; translation maps of DNA sequences were generated with the Sequence Manipulation Suite at [http://bioinformatics.org/ sms2/]. Multalin (5.4.1) at INRA [http:// prodes.toulouse.inra.fr/multalin/] (Corpet, 1988) and T-COFFEE (Version_1.41) [http:// www.ch.embnet.org/software/ TCoffee.html] (Notredame et al., 2000) were used for alignments production. Location of the potential plant promoter was computed by the TSSP program [http://www. softberry.com]. Phylogenies of OASTL and SAT isoforms were inferred by programs from the Phylip 3.36 package (Felsenstein, 2004).

\section{RESULTS AND DISCUSSION}

\section{Screening of the cDNA library of Nicotiana plumbaginifolia with the bacterial baits}

The $S$. cerevisiae strain PJ69-4, which has three reporter genes: HIS3, ADE2 and lacZ, was transformed with either pCE-GBT (containing cysE gene encoding SAT) or pCK-GBT (containing cysK gene encoding OASTL-A), respectively. Subsequently, both strains were transformed with the $N$. plumbaginifolia cDNA library and approximately $3 \times 10^{6}$ transformants were plated in each case. True positive prey clones, after re-transformation, were confirmed for their ability to activate the three reporter genes: HIS3, ADE2 and lac $Z$ when co-transforming yeasts with the respective baits. In summary, the two-hybrid screen of $N$. plumbaginifolia cDNA library resulted in three clones, two (Clone2 and Clone7) for the pCE-GBT bait and one (Clone6) for the pCK-GBT bait (Fig. 1). Bacterial SAT and OASTL were used as positive controls for the interactions. To our knowledge, this is the first demonstration using the two-hybrid system of interactions between bacterial enzymes SAT-SAT and SAT-OASTL. Interactions between bacterial OASTL monomers were undetectable in the two-hybrid system applied (not shown), probably because flexibility of the N-terminal part of the enzyme is a prerequisite for dimer formation (Burkhard et al., 1998). This is in agreement with the previously reported observation that interactions between plant OASTL monomers could not be demonstrated by a two-hybrid screen (Bogdanova \& Hell, 1997).

\begin{tabular}{|c|c|c|c|c|c|}
\hline Bait plasmid & Prey plasmid & SD-TL & SD-HTL & SD-ATL & $S D+X G A L$ \\
\hline pCE-GBT & pCK-GAD & 0 & 0 & 0 & 0 \\
\hline pCK-GBT & pCE-GAD & 0 & 3 & 0 & 0 \\
\hline pCE-GBT & pCE-GAD & 0 & $\mathrm{~s}$ & 0 & 0 \\
\hline pGBT (vector) & Clone 2 & 0 & & & 0 \\
\hline pGBT (vector) & Clone 6 & 0 & & & $\infty$ \\
\hline pGBT (vector) & Clone 7 & 0 & & & 0 \\
\hline pCE-GBT & Clone 2 & 0 & 3 & $\infty$ & 0 \\
\hline pCK-GBT & Clone 6 & 0 & 0 & 0 & 0 \\
\hline pCE-GBT & Clone 7 & 0 & 0 & 0 & 0 \\
\hline
\end{tabular}

Figure 1. Reconstitution of yeast two-hybrid interactions between the bacterial baits and the identified $N$. plumbaginifolia cDNA clones (preys).

Combinations of the plasmids transformed into a yeast reporter strain are indicated on the left. The cells were grown on a synthetic dropout medium lacking tryptophan and leucine (SD-TL), lacking histidine, tryptophan and leucine (SD-HTL) or lacking adenine, tryptophan and leucine (SD-ATL); positive interactions were also confirmed by $\beta$-galactosidase activity test (SD+XGAL).

\section{Characterization of the identified cDNAs}

The inserts of the identified clones were sequenced. Analysis of the sequences revealed that all three clones contain the deduced ORFs in frame with the activation domain of GAL4. The stop codons of the deduced ORFs were followed by non-coding sequences, terminating with poly(A) tracks. Clone6 appeared to be identical to Clone7. These clones contain ORF with a deduced amino-acid sequence having a strong similarity to serine acetyltransferases. Clone 2 contains an ORF with a deduced amino-acid sequence having a strong similarity to OASTL enzymes. The sequences of the $N$. plumbaginifolia cDNAs have been deposited in the GeneBank under the ac- 
cession numbers AY450296 (sat1) and AY450295 (cs1).

The closest known homologues (97\% and $77 \%$ identity, respectively) of $N$. plumbaginifolia NPSAT1 identified by us, encoded by Clone6 (and Clone7), are SAT4 and SAT1 from $N$. tabacum (Wirtz \& Hell, 2003), which are most probably a mitochondrial and a plastidic isoform of SAT, respectively. Since the cDNAs for $N$. tabacum SAT1 and SAT4 did not contain complete open reading frames it was difficult to evaluate the number of missing N-terminal codons in the N. plumbaginifolia cDNA isolated in this study (Fig. 2A). Comparison of $N$. plumbaginifolia SAT1 (NPSAT1) to the known plant SAT proteins revealed the strongest evolutionary homology to the organellar enzymes (Fig. 2B).

The closest homologue (94\% identity) of the $N$. plumbaginifolia CS1 (NPCS1) protein encoded
A

$$
\begin{aligned}
& \text { E.coli_SAT } \\
& \text { NPSAT1 } \\
& \text { N.tabacum_SAT } 4 \\
& \text { N.tabacum-SAT1 } \\
& \text { E.coli_SAT } \\
& \text { NPSAT1 } \\
& \text { N.tabacum SAT } 4 \\
& \text { N. tabacum_SAT1 } \\
& \text { E.coli_SAT } \\
& \begin{array}{l}
\text { E.coli_- } \\
\text { NPSAT1 }
\end{array} \\
& \frac{\text { NPSAT1 } 1}{\text { N.tabacum SAT } 4} \\
& \begin{array}{l}
\text { N.tabacum_SAT4 } \\
\text { N.tabacum_SAT1 }
\end{array} \\
& \text { E.coli_SAT } \\
& \text { NPSAT } 1 \\
& \text { N.tabacum_SAT } 4 \\
& \text { N.tabacum_SAT1 } \\
& \text { E.coli SAT } \\
& \text { NPSAT } 1 \\
& \text { N. tabacum SAT4 } \\
& \text { N. tabacum SAT } 1 \\
& \text { E.coli_SAT } \\
& \text { NPSAT1 } \\
& \begin{array}{l}
\text { N.tabacum_SAT4 } \\
N . \text { tabacum SAT } 1
\end{array}
\end{aligned}
$$

B

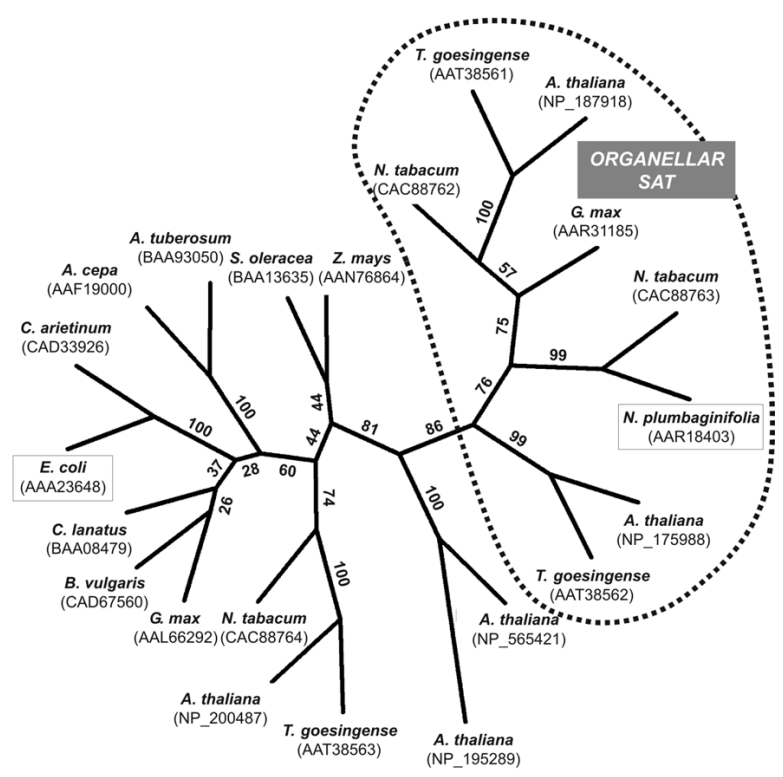

Figure 2. Amino-acid sequence alignment (A) and phylogenetic tree (B) of N. plumbaginifolia NPSAT1 and its selected homologues.

Panel A. Primary structure alignment of NPSAT1 (AAR18403) with $E$. coli SAT (AAA23648) and its two closest homologues, the organellar SAT4 (CAC88763) and SAT1 (CAC88762) from $N$. tabacum. The amino acids identical to those in NPSAT1 are highlighted in both sequences of $N$. tabacum proteins. Panel B. The twenty closest plant homologues of NPSAT1 with known (or at least presumed) intracellular location were selected after BLAST searches and the $E$. coli protein was included for comparison. The phylogenetic tree was constructed using full length proteins by the parsimony method and 100 bootstrap replicates (SEQBOOT, PROTPARS and CONSENSE of the Phylip v.3.63 program package). The GenBank accession numbers and the source organisms of the proteins used to generate the tree are included. The bootstrap values are given at the respective branches. 
by Clone2 is a cytosolic isoform of OASTL from Solanum tuberosum (Maruyama et al., 2001). Also in this case, the cDNA appeared to be incomplete and it could not be excluded that the protein is much longer at the $\mathrm{N}$-terminus. However, it is worth emphasizing that the sequence comparison to the closest homologues suggests that only two $\mathrm{N}$-terminal codons are missing in the identified cDNA (Fig. 3A). Comparison of the deduced ORF (NPCS1) to the known plant OASTL proteins including A. thaliana isoenzymes confirmed that it is located rather away from the organellar OASTL isoforms on the phylogenetic tree and revealed its strongest evolutionary homology to the cytosolic isoforms (Fig. 3B).

A.coli
NPCS1
C.tuberosum
C.vulgaris
E.coli
NPCS1
S.tuberosum
C. vulgaris

E.coli
NPCS1
S.tuberosum
C. vulgaris

E.coli
NPCS1
S.tuberosum
C. vulgaris

E.coli
NPCS1
S.tuberosum
C. vulgaris
E.coli
NPCS1
C. tublgaris

\begin{abstract}
--- MSK I FEDNSLT I GHTPLVRLNRIGNG---RI LAKVESRNPSFSVKCRIGANMIWDA 53 --GEKNGIAKDVTELIGNTPLVYLNNVVDGCVARVAAKLESMEPCSSVKDRIGYSMITDA 58 MAGEKIGIAKDVTELIGNTPLVYLNNVVDGCVARVAAKLESMEPCSSVKDRIGYSMITDA 60 MADAKSTIAKDVTELIGNTPLVYLNRVVDGCVARVAAKLEMMEPCSSVKDRIGYSMISDA 60

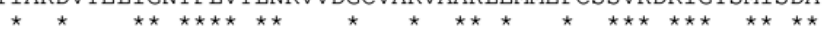

EKRGVLKPG-VELVEPTSGNTGIALAYVAAARGYKLTLTMPETMS IERRKLLKALGANLV 112 EEKGLIKPGESVLIEPTSGNTGVGLAFMAAAKGYKLI ITMPSSMS LERRI ILRAFGAELV 118 EEKGLIKPGESVLIEPTSGNTGVGLAFMAAAKGYKLI ITMPSSMSLERRI ILRGFRSELV 120 $\underset{*}{\text { ENKGLITPGESVLIEPTSGNTGIGLAFIAAAKGYRLI ICMPASMSLERRTI ILRAFGAELV }} 120$

LTEGAKGMKGAIQKAEEIVASNPEKYLLLQQFSNPANPEIHEKTTGPEIWEDTDGQVDVF 172 LTDPAKGMKGSIQKAEE IKAKTPN-SFILQQFENPANPKVHYETTGPEIWKGSNGKVDAL 177 LTDPAKGMKGAISKAEEIKAKT PN-SYILQQFENPANPKIHYETTGPEIWKGSNGKVDAL 179 LTDPARGMKGAVQKAEEIKAKTPN-SYILQQFENPANPKIHYETTGPEIWRGSGGKIDAL 179

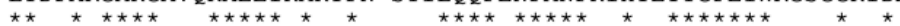

IAGVGTGGTLTGVSRYIKGTKGKTDLISVAVEPTDSPVIAQALAGEEIKPGPHKIQGIGA 232 VSGIGTGGTITGSGKYLR--EQNPDIKLYGVEPVESAILSGG------KPGPHKIQGIGA 229 ASGIGTGGTITGSGKYLR--EQNPNVKLYGVEPVESAILSGG------KPGPHKIQGIGA 231 VSGIGTGGTVTGAGKYLK--EQNPNIKLYGVEPVESAILSGG------KPGPHKIQGIGA 231

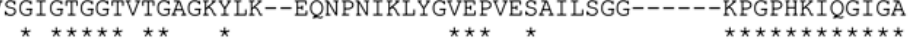

GFIPANLDLKLVDKVIGITNEEAISTARRLMEEEGILAGISSGAAVAAALKLQEDESFTN 292 GFIPGVLEVGLIDEVIQVSSEEAIETAKLLALKEGLLVGISSGASAAAAIKLAKRPENAG 289 GFI PGVLEVNLIDDVVOVSSEES IEMAKLLALKEGLLVGISSGAAAAAAIKVAKRPENAG 291 GFIPGVLDVNLLDEVIQVSSEES IETAKLLALKEGLLVGISSGAAAAAAIRIAKRPENAG 291

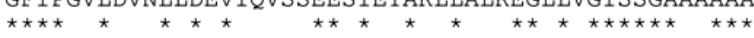

KNIVVILPSSGERYLSTALFADLFTEKELQQ--- 323 KLIVVVFPSFGERYLSSVLFESVRREAENMTVEP 323 KLIVVIFPSFGERYLSSVLFETVRREAENMTVEP 325 KLIVAVFPSFGERYLSTVLFESVKRETENMVFEP 325 $\star \star \star * * \star \star \star \star \star * \star * * * * *$
\end{abstract}

B

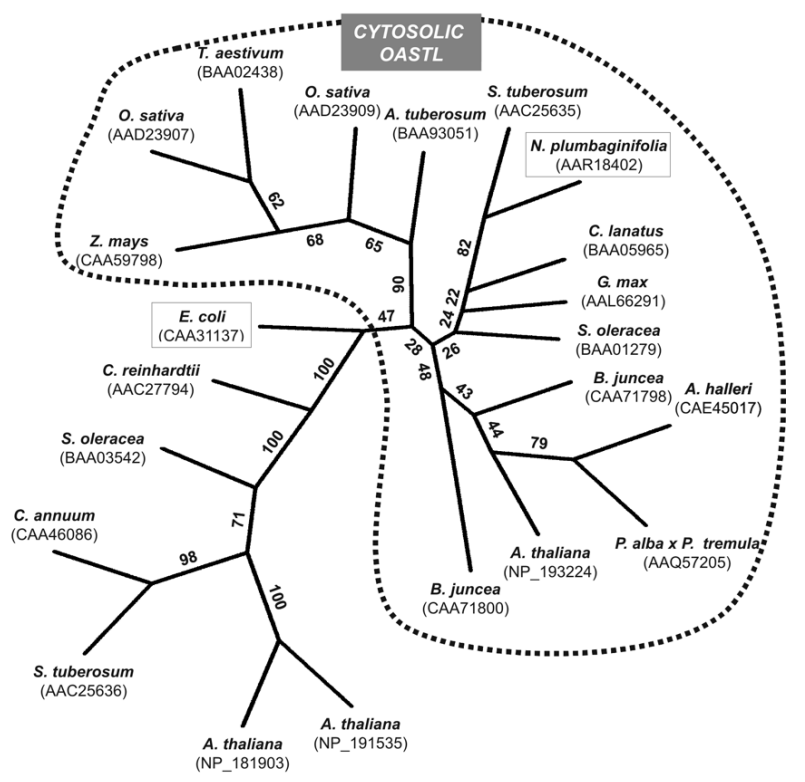

Figure 3. Amino-acid sequence alignment (A) and phylogenetic tree (B) of $N$. plumbaginifolia NPCS1 and its selected homologues.

Panel A. Primary structure alignment of NPCS1 (AAR18402) with $E$. coli OASTL-A (CAA31137) and its closest known homologues, the cytosolic isoforms of OASTL from potato ( $S$. tuberosum) and watermelon (C. vulgaris), AAC25635 and BAA05965, respectively. The stars indicate residues identical in all aligned proteins. Panel B. The twenty closest plant homologues of NPCS1 with known (or at least presumed) intracellular location were selected after BLAST searches and the $E$. coli protein was included for comparison. The phylogenetic tree was constructed using full length proteins by the parsimony method and 100 bootstrap replicates (SEQBOOT, PROTPARS and CONSENSE of the Phylip v.3.63 program package). The GenBank accession numbers and the source organisms of the proteins used to generate the tree are included. The bootstrap values are given at the respective branches. 
Enzymatic activity of OASTL in yeast cells transformed with the plasmids used in the two-hybrid analysis

The two-hybrid interactions indicated that despite the long $\mathrm{N}$-terminal extensions due to the presence of the activation domain of GAL4 protein, both the NPSAT1 and NPCS1 proteins retained the tertiary structure enabling interactions with other components of the cysteine synthase complex (Fig. 1 and Fig. 4A). In order to test whether such hybrid proteins still possess their catalytic properties we assayed the SAT and OASTL activity in yeast cells harboring the plasmids of interest. In $S$. cerevisiae endogenous SAT and OAS/OAH-TL activities are detectable, however, these enzymes were found not to constitute a cysteine biosynthetic pathway and L-cysteine is assumed to be exclusively synthesized through the cystathionine pathway (Ono et al., 1999). In none of the yeast transformants was the SAT activity increased in comparison to the control yeast strain transformed with empty vectors (not shown). The reason for this result is not clear, however, it cannot be excluded that the long N-terminal extension of SAT resulted in reduction of the enzymatic activity of this enzyme. Nevertheless, in the transformants harboring the plasmids that encoded either bacterial or plant OASTL, this enzymatic activity was increased 1.5 to 6 -fold in comparison to the controls (Fig. 4B). Hence, despite the reduced ability for the OASTL-OASTL dimer formation (due to the fusion at the $\mathrm{N}$-terminus), the enzyme is capable of catalyzing the reaction. It is necessary to note that the background OASTL activity observed in the yeast strain transformed with pCE-GAD and pGBT9 plasmids was defined as a background activity resulting from the yeast $O$-acetylserine/ $O$-acetylhomoserine sulfhydrylase (OAS/ OAH-TL) enzyme (Yamagata et al., 1974). In $S$. cerevisiae, impairment of the cystathionine pathway leads to cysteine auxotrophy (Brzywczy et al., 2002). Investigations on

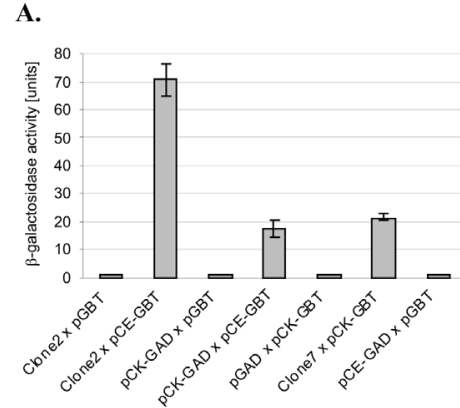

B.

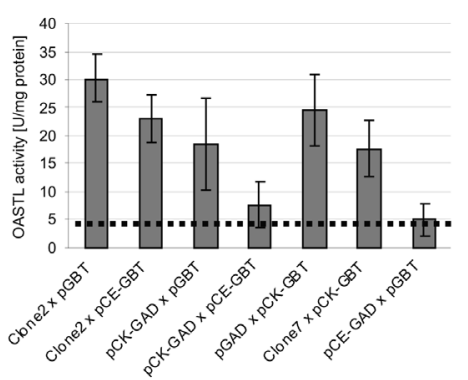

Figure 4. Enzymatic activities of yeast transformed with plasmids used in the two-hybrid analysis.

Each column represents an average value calculated from two independent colonies each assayed twice in triplicate with standard deviations indicated. The interactions monitored by $\beta$-galactosidase activity are in panel A, while the OASTL activity in panel B. The dotted vertical line indicates the background OASTL activity observed in the yeast strain.

cysteine auxotroph strains harboring separately or simultaneously the $E$. coli cys $E$ and cysK genes have been reported recently (Takagi et al., 2003); while yeast cells producing only exogenous SAT grew on a medium lacking L-cysteine, those producing only exogenous OASTL did not grow at all. The authors concluded (i) that $S$. cerevisiae SAT cannot support cysteine biosynthesis and has no metabolic role in vivo, and (ii) that $S$. cerevisiae OAS/OAH-TL produces L-cysteine provided that enough OAS is produced by $E$. coli SAT (Takagi et al., 2003). Expression of a plant SAT from sugar beet (Beta vulgaris) in $S$. cerevisiae took advantage of the OAS/ OAH-TL activity with OAS as substrate and introduced an alternative cysteine biosynthesis pathway in yeast (Mulet et al., 2004). 
OASTL is mostly active as a free enzyme, and a decrease of its catalytic activity while bound in a complex with SAT has been reported for both plant and $E$. coli enzymes
(Droux et al., 1998; Mino et al., 2000). In accordance with these data, we observed reduced activities of both OASTL enzymes (plant NPCS1 encoded by Clone2 and bacte-

A

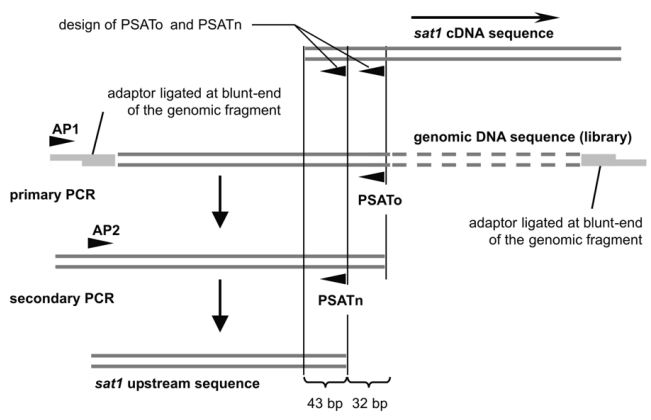

B

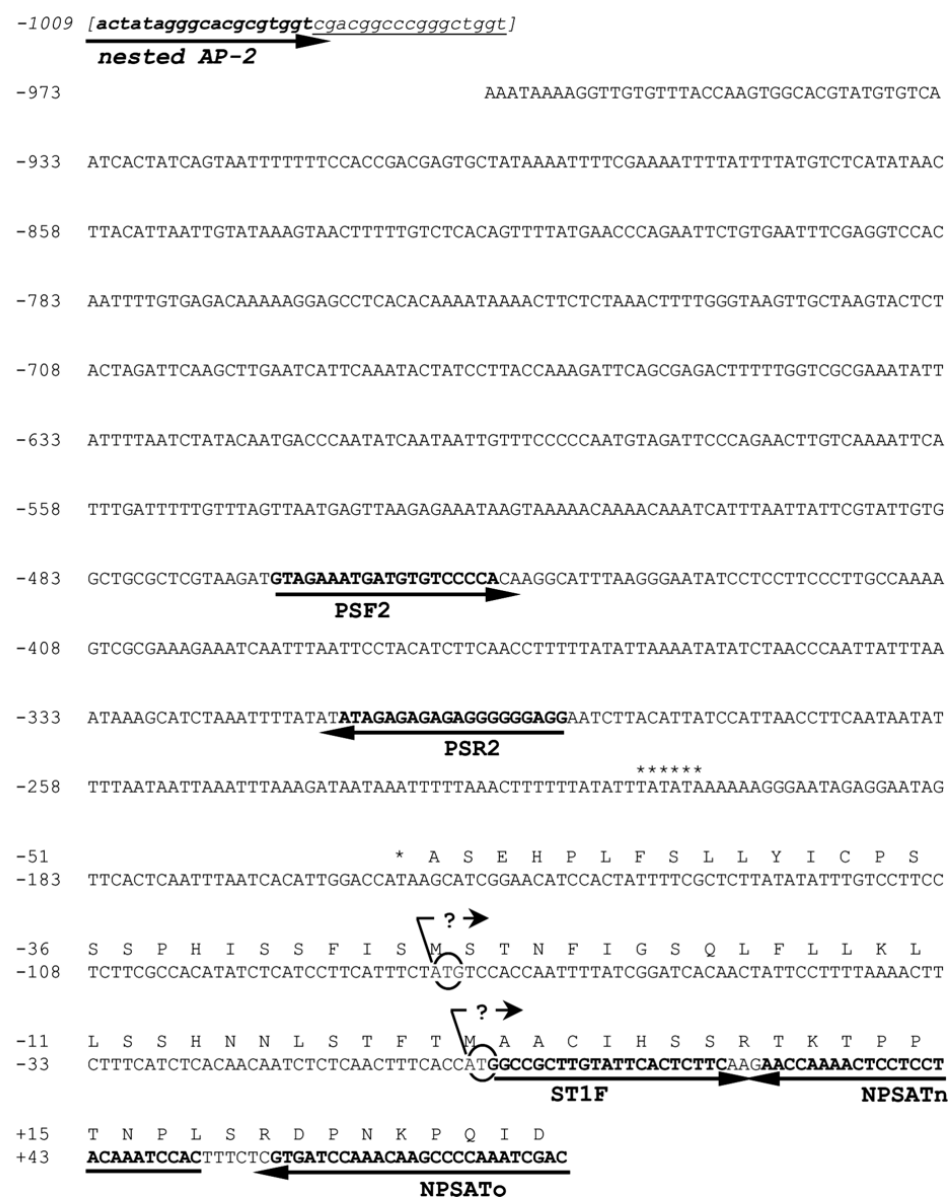

Figure 5. General scheme of the genome walking experiment (panel A) and the nucleotide sequence of the $N$. plumbaginifolia genomic DNA fragment upstream of sat1 (panel B).

Construction of the genomic library consisted of two steps: fragmentation of genomic DNA by a blunt-end restriction enzyme, followed by ligation of an adaptor to the ends of the DNA fragments. DNA was amplified from the library by two successive PCRs using an appropriate pair of oligonucleotides. AP1 and AP2 were complementary to the adaptor, PSATo (outer primer) and PSATn (nested primer) - complementary to the sat1 cDNA. Oligonucleotides used for cloning (AP2 and PSATn) and sequencing (PSF2 and PSR2) of the amplified genomic DNA fragment are indicated. The sequence corresponding to the GenomeWalker adaptor (removed) is shown in parentheses. The potential TATA-box and the potential transcription start point are indicated by stars. Translation of the DNA sequence in frame with the ORF encoding NPSAT1 revealed two possible "start" ATG codons (indicated by arrows). 
rial OASTL-A encoded by either pCK-GBT or pCK-GAD) in the presence of any isoform of SAT (either bacterial SAT encoded by pCE-GBT or plant NPSAT1 encoded by Clone7) in comparison to the controls without SAT (Fig. 4B). However, this effect was statistically significant $(P<0.05)$ only for the bacterial pair of the enzymes. The reduced OASTL activity in yeast overproducing simultaneously both SAT and OASTL isoenzymes corresponded to the significant increase of $\beta$-galactosidase activity in these strains, in comparison to the controls without SAT (Fig. 4A). Therefore, this system could be useful for an initial screening of the effects of targeted mutagenesis of OASTL on its activity and interaction with SAT. An advantage and simplicity of the system is that in the same cultures both OASTL activity can be assayed and interactions between OASTL and SAT monitored by $\beta$-galactosidase activity. Nevertheless, the results of such simple screening must be validated in other systems because an influence of yeast endogenous enzymes on the properties (both activities and interactions) of exogenous SAT and OASTL cannot be excluded. It is worth mentioning that no structural domains responsible for interactions of OASTL with SAT have been identified yet in the OASTL protein despite resolving of the crystal structures of bacterial SAT (Pye et al., 2004; Olsen et al., 2004) and OASTL-A (Burkhard et al., 1998).

\section{Genome walking upstream of sat1 se- quence}

The upstream genomic sequence of sat1 was revealed by genome walking. A general scheme of the experiment is shown in Fig. 5A. A DNA fragment of 999 bp was isolated from the $N$. plumbaginifolia genome that had a 43 bp overlap with the sat1 cDNA (Fig. 5B). The sequence was deposited in the GeneBank under the accession number AY898624. The nucleotide sequence (956 bp), identified as located upstream of the cloned cDNA, con- tained a potential plant promoter with a TATA-box upstream of the sequence encoding the N-terminal residues of the deduced protein including its potential signal peptide. However, experimental verification of the transcription start point should be performed in future in order to confirm the potential $5^{\prime}$ terminus of the Ntsat1 mRNA. Since the closest homologues of NPSAT1 (SAT4 and SAT1 from $N$. tabacum) were identified as partial cDNAs, we decided to make an alignment of NPSAT1 with their counterparts from $A$. thaliana; AtSAT1 (located to the mitochondria, the product of the intronless At3g13110 gene) and AtSAT3 (located to the chloroplasts, the product of the intronless At1g55920 gene). The alignment revealed a slightly higher percentage of identical residues between NPSAT1 and AtSAT1 than between NPSAT1 and AtSAT3 (Fig. 6A). The homology was rather poor in the N-terminal part of the proteins containing the signal peptides, which complicated the homology-based prediction of the intracellular location of NPSAT1. Additionally, computer analysis of the entire NPSAT1 protein failed to indicate the location of the mature product in one compartment with a high degree of certainty. In fact, plastidic, mitochondrial as well as secretory pathway-specific locations were suggested (Fig. 6B). Therefore, in silico analysis was not sufficient to answer the question about the intracellular location of NPSAT1 and additional experiments need to be conducted in order to solve this ambiguity.

\section{CONCLUSIONS}

In this study we demonstrated that the interactions between heterologous (bacterial- plant) components of the cysteine synthase complex are strong enough to enable cloning of the cDNA encoding plant enzymes using the bacterial enzymes as baits in the yeast two hybrid system. To our knowledge this is the first demonstration of application of this technology for 


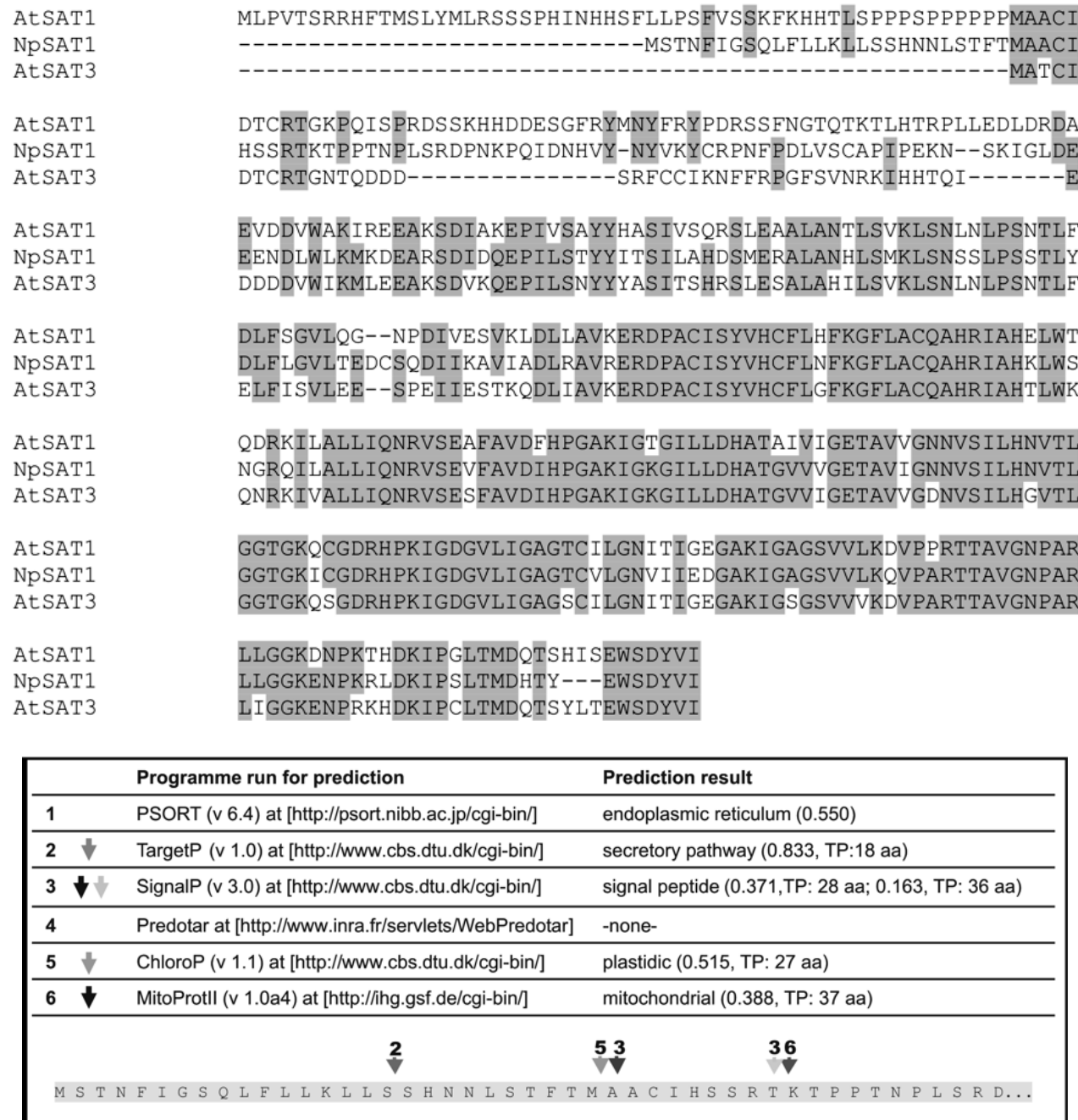

Figure 6. Alignement of the primary sequence of the longest deduced product of the sat1 gene with its closest homologues from A. thaliana (panel A) and a suggested intracellular location of the mature protein (panel B).

The residues identical between NPSAT1 and AtSAT1 and between NPSAT1 and AtSAT3 are highlighted. The suggested cleavage sites of the potential signal peptides are indicated and the locations of the mature protein according to the indicated computer programs are shown.

cloning of such cDNAs. The isolated clones contained almost complete open reading frames, as judged by sequence homologies as well as the 3 '-terminal non-coding sequences. Moreover, we propose that the two-hybrid approach could be a useful method for rapid verification of the effects of targeted mutagenesis of OASTL since it allows for uncomplicated monitoring of both the SAT-OASTL interactions and the enzymatic activity of OASTL in yeast cells transformed with the respective plasmids. In an attempt to obtain information about the nucleotide sequences located up- stream of the coding region of Npsat1 a genome walking experiment was performed and the potential transcription and translation starts were identified by computer analysis. The deduced full-length ORF of NPSAT1 has a slightly stronger homology to the mitochondrial than to the chloroplastic SAT isoenzymes. Thus, this protein is envisaged to be located rather in mitochondria. However, the programs predicting intercellular locations by analysis of signal peptides failed to localize NPSAT1 to any cellular compartment with a high degree of confidence. 


\section{R E F E R E N C E S}

Berkowitz O, Wirtz M, Wolf A, Kuhlmann J, Hell R. (2002) Use of biomolecular interaction analysis to elucidate the regulatory mechanism of the cysteine synthase complex from Arabidopsis thaliana. J Biol Chem.; 277: 30629-34.

Blaszczyk A, Brodzik R, Sirko A. (1999) Increased resistance to oxidative stress in transgenic tobacco plants overexpressing bacterial serine acetyltransferase. Plant J.; 20: $237-43$.

Blaszczyk A, Sirko L, Hawkesford MJ, Sirko A. (2002) Biochemical analysis of transgenic tobacco lines producing bacterial serine acetyltransferase. Plant Sci.; 162: 589-97.

Bogdanova N, Hell R. (1997) Cysteine synthesis in plants: protein-protein interactions of serine acetyltransferase from Arabidopsis thaliana. Plant J.; 11: 251-62.

Brzywczy J, Sienko M, Kucharska A, Paszewski A. (2002) Sulphur amino acid synthesis in Schizosaccharomyces pombe represents a specific variant of sulphur metabolism in fungi. Yeast.; 19: 29-35.

Burkhard P, Rao GS, Hohenester E, Schnackerz KD, Cook PF, Jansonius JN. (1998) Three-dimensional structure of $O$-acetylserine sulfhydrylase from Salmonella typhimurium. J Mol Biol.; 283: 121-33.

Corpet F. (1988) Multiple sequence alignment with hierarchical clustering. Nucleic Acids Res.; 16: 10881-90.

Droux M, Ruffet ML, Douce R, Job D. (1998) Interactions between serine acetyltransferase and $O$-acetylserine (thiol) lyase in higher plants - structural and kinetic properties of the free and bound enzymes. Eur $J$ Biochem.; 255: 235-45.

Felsenstein J. (2004) PHYLIP (Phylogeny Inference Package) version 3.6. Distributed by the author. Department of Genome Sciences, University of Washington, Seattle.

Fields S, Song O. (1989) A novel genetic system to detect protein-protein interactions. $N a$ ture.; 340: 245-6.
Gaitonde MK. (1967) A spectrophotometric method for the direct determination of cysteine in the presence of other naturally occurring amino acids. Biochem J.; 104: 627-33.

Harms K, von Ballmoos P, Brunold C, Hofgen R, Hesse H. (2000) Expression of a bacterial serine acetyltransferase in transgenic potato plants leads to increased levels of cysteine and glutathione. Plant J.; 22: 335-43.

Hell R, Jost R, Berkowitz O, Wirtz M. (2002) Molecular and biochemical analysis of the enzymes of cysteine biosynthesis in the plant Arabidopsis thaliana. Amino Acids.; 22: $245-57$.

Hindson VJ, Shaw W. (2003) Random-order ternary complex reaction mechanism of serine acetyltransferase from Escherichia coli. Biochem J.; 42: 3113-9

Howarth JR, Roberts MA, Wray JL. (1997) Cysteine biosynthesis in higher plants: a new member of the Arabidopsis thaliana serine acetyltransferase small gene-family obtained by functional complementation of an Escherichia coli cysteine auxotroph. Biochim Biophys Acta.; 1350: 123-7.

James P, Halladay J, Craig EA. (1996) Genomic libraries and a host strain designed for highly efficient two-hybrid selection in yeast. Genetics.; 144: 1425-36.

Jost R, Berkowitz O, Wirtz M, Hopkins L, Hawkesford MJ, Hell R. (2000) Genomic and functional characterization of the oas gene family encoding $O$-acetylserine (thiol) lyases, enzymes catalyzing the final step in cysteine biosynthesis in Arabidopsis thaliana. Gene; 253: $237-47$.

Kredich NM. (1996) Biosynthesis of cysteine. In Escherichia coli and Salmonella typhimurium. Cellular and Molecular Biology. Neidhardt FC, Curtiss R, Ingraham JL, Linn ECC, Low KB, Magasanik B, Reznikoff WS, Riley M, Schaechter M, Umberger M, eds, pp 514-27. ASM Press, Washington DC.

Kuske CR, Hill KK, Guzman E, Jackson PJ. (1996) Subcellular location of $O$-acetylserine sulfhydrylase isoenzymes in cell cultures and 
plant tissues of Datura innoxia Mill. Plant Physiol.; 112: 659-67.

Leustek T, Saito K. (1999) Sulfate transport and assimilation in plants. Plant Physiol.; 120: $637-44$.

Liszewska F, Blaszczyk A, Sirko A. (2001) Modification of non-protein thiols contents in transgenic tobacco plants producing bacterial enzymes of cysteine biosynthesis pathway. Acta Biochim Polon.; 48: 647-56.

Maruyama A, Saito K, Ishizawa K. (2001) Beta-cyanoalanine synthase and cysteine synthase from potato: molecular cloning, biochemical characterization, and spatial and hormonal regulation. Plant Mol Biol.; 46: 749-60.

Marzluf GA. (1997) Molecular genetics of sulfur assimilation in filamentous fungi and yeast. Annu Rev Microbiol.; 51: 73-96.

Mino K, Yamanoue T, Sakiyama T, Eisaki N, Matsuyama A, Nakanishi K. (2000) Effects of bienzyme complex formation of cysteine synthetase from Escherichia coli on some properties and kinetics. Biosci Biotechnol Biochem.; 64: 1628-40.

Mulet J-M, Alemany B, Ros R, Calvete J, Serrano R. (2004) Expression of a plant serine $O$-acetyltransferase in Saccharomyces cerevisiae confers osmotic tolerance and creates an alternative pathway for cysteine biosynthesis. Yeast.; 21: 303-12.

Noji M, Murakoshi I, Saito K. (1994) Molecular cloning of a cysteine synthase cDNA from Citrullus vulgaris (watermelon) by genetic complementation in an Escherichia coli Cysauxotroph. Mol Gen Genet.; 244: 57-66.

Noji M, Inoue K, Kimura N, Gouda A, Saito K. (1998) Isoform-dependent differences in feedback regulation and subcellular localization of serine acetyltransferase involved in cysteine biosynthesis from Arabidopsis thaliana. J Biol Chem.; 273: 32739-45.

Notredame C, Higgins D, Heringa J. (2000) T-Coffee: A novel method for multiple sequence alignments. $J$ Mol Biol.; 302: 205-17.
Olsen L, Hug B, Vetting M, Roderick S. (2004) Structure of serine acetyltransferase in complexes with CoA and its cysteine feedback inhibitor. Biochemistry.; 43: 6013-9.

Ono B-I, Hazu T, Yoshida S, Kawato T, Shinoda S, Brzywczy J, Paszewski A. (1999) Cysteine biosynthesis in Saccharomyces cerevisiae: a new outlook on pathway and regulation. Yeast.; 15: 1365-75.

Pye V, Tingley A, Robson R, Moody P. (2004) The structure and mechanism of serine acetyltransferase from Escherichia coli. J Biol Chem.; 279: 40729-36.

Saito K, Yokoyama H, Noji M, Murakoshi I. (1995) Molecular cloning and characterization of a plant serine acetyltransferase playing a regulatory role in cysteine biosynthesis from watermelon. $J$ Biol Chem.; 270: 16321-6.

Sambrook J, Frisch EF, Maniatis T. (1989) Molecular Cloning: A Laboratory Manual. Cold Spring Harbor Laboratory Press, Cold Spring Harbor.

Sirko A, Błaszczyk A, Liszewska F. (2004) Overproduction of SAT and/or OASTL in transgenic plants: a survey of effects. J Exp Bot.; 55: $1881-8$.

Tai CH, Burkhard P, Gani D, Jenn T, Johnson C, Cook PF. (2001) Characterization of the allosteric anion-binding site of O-acetylserine sulfhydrylase. Biochemistry.; 40: 7446-52.

Takagi H, Yoshioka K, Awano N, Nakamori S, Ono B. (2003) Role of Saccharomyces cerevisiae serine $O$-acetyltransferase in cysteine biosynthesis. FEMS Microbiol Lett.; 218: 291-7.

Wirtz M, Hell R. (2003) Production of cysteine for bacterial and plant biotechnology: application of cysteine feedback-insensitive isoforms of serine acetyltransferase. Amino Acids.; 24: 195-203.

Yamagata S, Takeshima K, Naiki N. (1974) Evidence for the identity of $O$-acetylserine sulfhydrylase with $O$-acetylhomoserine sulfhydrylase in yeast. $J$ Biochem.;

75: 1221-9. 